\title{
Molecular cloning and characterization of an inhibitor of apoptosis protein (IAP) from the tiger shrimp, Penaeus monodon is
}

\author{
Jiann-Horng Leu, Yu-Chen Kuo, Guang-Hsiung Kou*, Chu-Fang Lo*
}

Institute of Zoology, National Taiwan University, Taipei 10617, Taiwan, ROC

Received 15 March 2007; received in revised form 19 April 2007; accepted 7 May 2007

Available online 14 June 2007

\section{KEYWORDS}

Penaeus monodon;

Inhibitor of apoptosis

protein;

IAP;

Reaper;

White spot syndrome

virus;

WSSV;

Apoptosis;

WSSV449

\begin{abstract}
The inhibitor of apoptosis proteins (IAPs) play important roles in both apoptosis and innate immunity. Here, we report the first cloning and characterization of a novel IAP family member, PmIAP, from Penaeus monodon. The full-length PmIAP cDNA is $4769 \mathrm{bp}$, with an ORF encoding a protein of 698 amino acids. The PmIAP protein contains three BIR domains and a C-terminal RING domain, and its mRNA was expressed in all analyzed tissues. In insect cells, PmIAP, together with Spodoptera frugiperda IAP, AcMNPV P35, and WSSV449 (or ORF390, an anti-apoptosis protein encoded by white spot syndrome virus), could all block the apoptosis induced by Drosophila Reaper protein (Rpr), whereas only P35 and WSSV449 could block the apoptosis induced by actinomycin D. Co-immunoprecipitation showed that PmIAP physically interacted with Rpr, and in an immunofluorescent analysis the two proteins produced co-localized punctate signals in the cytoplasm. Deletion analysis revealed that both the BIR2 and BIR3 domains of PmIAP could independently bind to and inhibit Rpr, whereas the BIR1 domain could not. These results strongly suggest that PmIAP blocks Rpr's pro-apoptotic activity through mechanisms that are evolutionarily conserved across crustaceans, insects, and mammals.

(c) 2007 Elsevier Ltd. All rights reserved.
\end{abstract}

Abbreviations: IAP, inhibitor of apoptosis protein; ORF, open reading frame; ActD, actinomycin D; WSSV, white spot syndrome virus; BIR, baculoviral IAP repeat; Rpr; reaper; IBM, IAP-binding motif; UTR, untranslated region.

GenBank accession number of Penaeus monodon inhibitor of apoptosis protein: EF114675.

*Corresponding authors. Tel.: +886223633562; fax: +886223638179 .

E-mail addresses: ghkou@ntu.edu.tw (G.-H. Kou), gracelow@ntu.edu.tw (C.-F. Lo).

\section{Introduction}

Apoptosis is a genetically programmed cellular suicide process that eliminates unwanted or diseased cells, and it plays important roles in embryogenesis, homeostasis, insect metamorphosis, and immunity [1-3]. Cells undergoing apoptosis display a series of morphological changes, including cell shrinkage, chromatin condensation, DNA fragmentation, membrane blebbing, and, finally, formation of 
apoptotic bodies [4]. The concept of apoptosis was first introduced in 1972 [5]. Since then, hundreds of genes that control apoptosis have been identified in different species, which is good evidence that the mechanisms and the molecules involved in this suicide program have been highly conserved throughout metazoan evolution [6].

Inhibitor of apoptosis proteins (IAPs) are a conserved group of proteins that regulate apoptosis in both vertebrates and invertebrates. Since the first discovery of an IAP gene in a baculovirus [7], numerous cellular IAP homologs have been identified [8,9]. The hallmark of an IAP protein is the presence of one to three copies of a zinc-binding baculoviral IAP repeat (BIR) domain. These BIR domains bind directly to the caspases to inhibit them, and they are essential for the anti-apoptotic properties of the IAPs $[10,11]$. The interaction between BIR domains and caspases is negatively regulated by proteins that contain an IAP-binding motif (IBM), including Drosophila Reaper (Rpr), Grim, and Hip, and mammalian Smac/DIABLO. The only sequence homology shared by these IBM proteins is a short consensus sequence at their amino termini. This conserved sequence is the IBM, which is responsible for binding to the BIR domain and interfering with IAP-mediated caspase inhibition [12]. The IAPs also contain another zinc-binding domain known as the RING domain, which is invariably located at their C-termini. The RING domain has an ubiquitin E3 ligase activity, which attaches ubiquitin to proteins that bind to the IAPs, including caspases, some IBM proteins, and IAP itself. The ubiquitinated IAP and IBM proteins are then subject to proteasomal degradation, while the ubiquitinated caspases, instead of being degraded, become inactive [12-14].

In addition to acting as important regulators in apoptosis, recent evidence suggests that some IAPs also play important roles in innate immunity in both mouse and Drosophila [15-19]. In Drosophila, microbe infection initiates innate immune responses through two major, distinct signaling pathways: the Toll pathway and the immune deficiency (Imd) pathway. The Toll pathway is predominantly activated by fungal and Gram-positive bacterial infections, whereas the Imd pathway responds to infection by Gram-negative bacteria. The activation of these two pathways causes members of the nuclear factor kB (NF-kB) family to translocate to the nucleus to activate the transcription of antimicrobial peptide genes [20]. The importance of Drosophila IAP2 (DIAP2) in Drosophila innate immunity was first revealed by two independent studies using large-scale RNAi screening in cultured Drosophila cells to identify novel components in the Imd pathway. Both studies showed that diap2 RNAi abrogated the Imd-signaling antimicrobial peptide response $[16,17]$. Moreover, diap2 null mutant Drosophila fail to induce the synthesis of antimicrobial peptides and are highly susceptible to infection by Gram-negative bacteria $[18,19]$.

In the present paper, based on annotations of a $5^{\prime}$ EST database generated from a Penaeus monodon post-larvae cDNA library, we identified a cDNA clone that appeared to encode an IAP protein. Since no crustacean IAP has been reported, we therefore decided to clone the full-length CDNA sequence of this $P$. monodon IAP (PmIAP) and to characterize this protein. In a series of functional assays, other anti-apoptosis proteins were also included for comparison. One of these is a novel, recently reported [21], anti-apoptosis protein (WSSV449, or ORF390) encoded by a shrimp viral pathogen, white spot syndrome virus (WSSV).

\section{Materials and methods}

\subsection{Identification of a cDNA clone of a $P$. monodon inhibitor of apoptosis protein (PmIAP)}

A cDNA library constructed from WSSV-infected postlarvae of $P$. monodon was subjected to large-scale $5^{\prime}$ and $3^{\prime}$ end sequencing, and the sequencing results were used to generate a $5^{\prime}$ and $3^{\prime}$ EST database. Analysis of the $5^{\prime}$ EST database using BLASTX against the NCBI non-redundant (nr) database revealed that one cDNA clone, PmTwI09F1, contained a partial nucleotide sequence that was highly homologous to the RING domain of the Bombyx mori inhibitor of apoptosis protein. Complete sequencing of this clone by primer walking showed that it contained only part of the nucleotide sequence of a putative $P$. monodon IAP gene.

\subsection{Isolating the $5^{\prime}$ end of PmIAP CDNA}

Pleopod (swimming leg) mRNA was purified using a QuickPrep Micro mRNA Purification Kit according to the manual supplied by the manufacturer (GE Healthcare). Briefly, pleopods from adult shrimp were powdered in the presence of liquid nitrogen, and further homogenized in the extraction buffer supplied with the kit. The homogenate was diluted with elution buffer, clarified by centrifugation at $12000 \mathrm{~g}$ for $5 \mathrm{~min}$, and then mixed with oligo(dT)-cellulose. After extensive washing with high- and low-salt buffers, the mRNA was eluted with elution buffer, precipitated with ethanol, and then quantified at A260. A 5' RACE kit was then used to isolate the $5^{\prime}$ end of PmIAP CDNA according to the instructions provided by the manufacturer (Roche Molecular Biochemicals). Three gene-specific primers, I09F1-SP1, I09F1-SP2, and I09F1-SP3 (Table 1), were designed according to the nucleotide sequence of the PmTwI09F1 cDNA clone. The first-strand cDNA was synthesized from $100 \mathrm{ng}$ pleopod mRNA using 109F1-SP1 primer, tailed at the $3^{\prime}$ end with dATPs using the terminal transferase, and then subjected to firstround PCR using oligo(dT)-anchor primer and I09F1-SP2 primer. The PCR product was diluted and subjected to second-round PCR using anchor primer and I09F1-SP3 primer. The final PCR products of $5^{\prime}$ RACE were cloned into the PGEM-T easy vector (Promega) and used to obtain the complete cDNA sequence of PmIAP.

\subsection{Sequence analysis of PmIAP}

The deduced amino acid sequence of PmIAP was analyzed with BLASTP against the NCBI nr database and ScanProsite. For multiple sequence alignment, the BIR1, 2, and 3 domains and RING domain from PmIAP were aligned using GeneDoc (ver. 2.6.002) with the corresponding domains from SfIAP (AX213188), DIAP1 (Q24306), DIAP2 (Q24307), and XIAP (P98170). 
Table 1 Primer sequence list for the $5^{\prime}$ RACE and RT-PCR

\begin{tabular}{lll}
\hline Primer name & Primer sequence & Usage \\
\hline I09F1-SP1 & $5^{\prime}$-TCACACTTCAGGATACAATAGGT-3' & IAP 5' RACE \\
I09F1-SP2 & $5^{\prime}$-CTGAGTAAGAGCGACTCACAAG-3' & IAP 5' RACE \\
I09F1-SP3 & $5^{\prime}$-ACGACGTCCATCTCAGCATCCA-3' & IAP 5' RACE \\
109F01R1 & $5^{\prime}$-AGAAGAATAACGGCAAGTGACTG-3' & IAP RT-PCR \\
109F01F1 & $5^{\prime}$-GCCTCTTTAGGTCAAGTGTAG-3' & IAP RT-PCR \\
P1882-actin F1 & $5^{\prime}$-CCGTCATCAGGGTGTGATGGT-3' & Actin RT-PCR \\
P1883-actin R1 & $5^{\prime}$-CCACGCTCAGTCATGATCTTCA-3' & Actin RT-PCR \\
\hline
\end{tabular}

\subsection{Tissue distribution of PmIAP mRNA}

Total RNAs were extracted from various tissues using TRIzol reagent (Invitrogen, USA) according to the procedure provided by the supplier. After quantification at A260, about $10 \mu \mathrm{g}$ of total RNAs were treated with DNase I, extracted with phenol-chloroform, and then precipitated with ethanol. The DNase I-treated total RNAs were primed with oligo-dT-anchor primer (Roche) and reverse-transcribed with SuperScript II (Invitrogen, USA) at $42^{\circ} \mathrm{C}$ for $50 \mathrm{~min}$. Aliquots of this CDNA were then used for PCR analysis. The PmIAP primer pair used for RT-PCR was 109F01R1 and 109F01F1 (Table 1). For internal control, an actin gene primer pair, P1882-actin F1 and P1883-actin R1 (Table 1), was designed based on $P$. monodon actin 2 (AF100987).

\subsection{Plasmid construction}

The pilot study showed that the insect cell expression vector, pIZ/V5-His (Invitrogen), was apparently unable to express any of the desired proteins in sufficient quantities (data not shown). We followed Clem and Miller [22] and designed expression constructs based on the promoter of the Drosophila heat shock protein 70 (hsp70) gene. The Drosophila melanogaster hsp70 promoter region (from -525 to +236 , according to Torok and Karch [23], and GenBank accession no. J01103) was PCR amplified from D. melanogaster genomic DNA using forward primer 5'CCTCATGAGTTGACAACAACAGTCTTGACAACCT-3' and reverse primer 5'-GGAAGCTTCCTCGGTAACGACTTGTTGAAAGT-3' (underlined nucleotides correspond to cutting sites for $\mathrm{BspHI}$ and HindIII, respectively), and the PCR product was cloned into PGEM-T easy vector. To construct the heat-inducible expression vector, the $\mathrm{plZ/V5-His} \mathrm{plasmid} \mathrm{(Invitrogen)} \mathrm{was}$ digested with $\mathrm{BspHI}$ and HindIII to release the OPIE2 promoter and then ligated with the $\mathrm{BspHI}$ and HindlIIdigested hsp70 DNA fragment. The resulting plasmid was named $\mathrm{pDHsp} / \mathrm{V} 5$-His. A second heat-inducible expression vector, $\mathrm{pDHsp} / \mathrm{FLAG}-\mathrm{His}$, was constructed by digesting the pDHsp/V5-His with Sacll and Mlul to release the V5 tag and ligated with the FLAG tag fragment (5'-GGGACTACAAAGACGATGACGACAAGA-3' and 5'-CGCGTCTTGTCGTCATCGTCTTTGTAGTCCCGC-3').

The genes analyzed in this study include Drosophila rpr (NM_079414), PmIAP (EF114675), SfIAP (AX213188), AcMNPV p35 (P08160), and wssv449 (NP_477912). These genes were inserted into $\mathrm{pDHsp} / \mathrm{V} 5-\mathrm{His}$ or $\mathrm{pDHsp} / \mathrm{FLAG}-\mathrm{His}$ by $\mathrm{PCR}$ cloning. The primers used for PCR and the corresponding constructs are listed in Table 2.

Various PmIAP deletion constructs were constructed by PCR using the plasmid pDHsp-PmIAP/V5 as template. The amplified fragments were cloned into $\mathrm{pDHsp}-\mathrm{His} / \mathrm{V} 5$ to generate the corresponding expression constructs, all of which produced a V5-tagged fusion protein. The constructs and the used primers are listed in Table 3.

\subsection{Cell culture}

Spodoptera frugiperda (Lepidoptera: Noctuidae) SF-9 insect cells were maintained at $27^{\circ} \mathrm{C}$ in Sf-900 II SFM medium (Invitrogen) supplemented with $10 \%$ heat-inactivated fetal bovine serum (Hyclone Laboratories).

\subsection{Actinomycin D assay}

SF-9 cells seeded in six-well plates $\left(1 \times 10^{6}\right.$ cells/well) were transfected with $4 \mu \mathrm{g}$ of the constructed plasmids by Cellfectin reagent (Invitrogen) according to the procedure described by the supplier. At $18-20 \mathrm{~h}$ post-transfection, the cells were heat shocked in a $42^{\circ} \mathrm{C}$ water bath for 30 min to induce the expression of the transfected genes placed under the hsp70 promoter, and then returned to $27^{\circ} \mathrm{C}$. At $4 \mathrm{~h}$ after heat shock, the media were removed and replaced with fresh media containing $150 \mathrm{ng} / \mathrm{ml}$ of actinomcyin D (ActD). At $14-16 \mathrm{~h}$ after addition of ActD, the media was removed and the cells were washed with phosphate-buffered saline (PBS; $137 \mathrm{mM} \mathrm{NaCl}, 10 \mathrm{mM}$ phosphate, $2.7 \mathrm{mM} \mathrm{KCl}, \mathrm{pH} 7.4$ ) and then stained with $0.04 \%$ trypan blue in PBS. The viable, intact cells were counted using a bright-field microscope equipped with a digital camera and AutoCell software. The total number of viable cells was determined from five evenly distributed fields of view at $200 \times$ magnification. The viability of the cells used to express the indicated proteins was calculated by dividing the total number of viable cells remaining after drug treatment by the total number of viable cells in control transfections at the time of drug addition. Results were expressed as mean \pm S.D.; $n=3$.

\subsection{Viability assays for Rpr-induced apoptosis}

SF-9 cells seeded in six-well plates $\left(5 \times 10^{5}\right.$ cells/well) were transfected with the appropriate plasmids using Cellfectin reagent (Invitrogen). After transfection for $16-18 \mathrm{~h}$, the cells were heat shocked in a $42{ }^{\circ} \mathrm{C}$ water bath for $30 \mathrm{~min}$ and 
Table 2 Primer sequence list for the construction of various expression plasmids

\begin{tabular}{|c|c|c|c|c|}
\hline Gene & Primer sequence & Cutting site & Vector & Tag \\
\hline \multirow[t]{2}{*}{ Rpr } & F: 5'-GGAAGCTTACCATGGCAGTGGCATTCTACATACCCGA-3' & HindIII & $\mathrm{pDHsp} / \mathrm{V} 5-\mathrm{His}$ & No \\
\hline & R: $5^{\prime}$-TCGGATCCTCATTGCGATGGCTTGCGA-3' & BamHI & & \\
\hline \multirow[t]{2}{*}{ Rpr } & F: 5'-GGAAGCTTACCATGGCAGTGGCATTCTACATACCCGA-3' & HindIII & $\mathrm{pDHsp} / \mathrm{FLAG}-\mathrm{His}$ & Flag \\
\hline & R: 5'-CGAACCGCGGTTGCGATGGCTTGCGATATTTG-3' & Sacll & & \\
\hline \multirow[t]{2}{*}{ PmIAP } & F: 5'-GAAGCTTACCATGGGTGATAGTCCCACGATCAG-3' & HindIII & $\mathrm{pDHsp} / \mathrm{V} 5-\mathrm{His}$ & V5 \\
\hline & R: 5'-AGCGGCCGCCGGATACAATAGGTTTGAGGTGAATTTG-3' & Notl & & \\
\hline \multirow[t]{2}{*}{ SfIAP } & F: 5'-GAAGCTTACCATGTGGTCGTGTTCCTTACCT- $3^{\prime}$ & HindIII & $\mathrm{pDHsp} / \mathrm{V} 5-\mathrm{His}$ & V5 \\
\hline & R: 5'-GCAGAATTCCACGAGAAATATAACCGCACTGCATTTTG -3' & EcoRI & & \\
\hline \multirow[t]{2}{*}{ AcMNPV p35 } & F: 5'-TACCATGTGTGTAATTTTTCCGGTAGAAATC-3' & & $\mathrm{pDHsp} / \mathrm{V} 5-\mathrm{His}$ & V5 \\
\hline & R: 5'-GGTGGATCCTTTAATTGTGTTTAATATTACATTTTTGTTGAGTG-3' & BamHI & & \\
\hline \multirow[t]{2}{*}{ Wssv449 } & F: 5'-CGGAAGCTTATGTGCACATTAAAAACATACA-3' & HindIII & $\mathrm{pDHsp} / \mathrm{V} 5-\mathrm{His}$ & V5 \\
\hline & R: $5^{\prime}$-GCCGGATCCTACTCCACGCTGCTTGGA-3' & BamHI & & \\
\hline
\end{tabular}

Table 3 Primer sequence list for the preparation of various PmIAP deletion constructs

\begin{tabular}{|c|c|c|c|}
\hline Construct & Primer sequence & Cutting site & Region (aa) \\
\hline \multirow[t]{2}{*}{ BIR } & F: 5'-CTGCAACTACTGAAATCAACCAAG-3' & & $1-358$ \\
\hline & R: 5'-AGCGGCCGCCACTTGCCGATACTGTGCTAGTACTTG-3' & Notl & \\
\hline \multirow[t]{2}{*}{ RING } & F: 5'-GAAGCTTACCATGAGTACTAGCACAGTATCGGCAAG-3' & HindIII & $351-698$ \\
\hline & R: 5'-AGCGGCCGCCGGATACAATAGGTTTGAGGTGAATTTG-3' & Notl & \\
\hline \multirow[t]{2}{*}{ BIR1 } & F: 5'-CTGCAACTACTGAAATCAACCAAG-3' & & $1-92$ \\
\hline & R: 5'-GTTGCGGCCGCCTCTAGTGGGCCTGGGAATGGT-3' & Notl & \\
\hline \multirow[t]{2}{*}{ BIR2 } & F: 5'-GGTAAGCTTACCATGACCATTCCCAGGCCCACTAGAA- $3^{\prime}$ & HindIII & $86-227$ \\
\hline & R: 5'-GTTGCGGCCGCCACGACATTCTGGATAGGAACCA-3' & Notl & \\
\hline \multirow[t]{2}{*}{ BIR3 } & F: 5'-GGTAAGCTTACCATGGACGTGTCCTGGGAGGAGATTG-3' & HindIII & $233-358$ \\
\hline & R: 5'-AGCGGCCGCCACTTGCCGATACTGTGCTAGTACTTG-3' & Notl & \\
\hline \multirow[t]{2}{*}{ BIR1+2 } & F: 5'-CTGCAACTACTGAAATCAACCAAG-3' & & $1-193$ \\
\hline & R: 5'-GTTGCGGCCGCCTATGCTTGGTAGACAGGCTAGGA-3' & Notl & \\
\hline \multirow[t]{2}{*}{$\mathrm{BIR} 2+3$} & F: 5'-GGTAAGCTTACCATGACCATTCCCAGGCCCACTAGAA-3' & HindIII & $86-358$ \\
\hline & R: 5'-AGCGGCCGCCACTTGCCGATACTGTGCTAGTACTTG-3' & Notl & \\
\hline
\end{tabular}

then returned to $27^{\circ} \mathrm{C}$. At $5 \mathrm{~h}$ after heat shock, the viability of the SF-9 cells was determined according to Clem and Miller [22]. Briefly, the media were removed, and the cells were resuspended in $1 \mathrm{ml}$ PBS containing 0.04\% trypan blue with a rubber policeman. The viable intact cells were counted with a hemocytometer, and eight grids were counted for each sample. The mean and standard deviation were calculated from three replicate samples. Relative percent viability was calculated by comparing the number of viable cells transfected with various expression plasmids with the number of viable cells transfected with the control plasmid. The relative percent viability for cells transfected with the control plasmid was set at $100 \%$.

\subsection{Immunoblot analysis}

SF-9 cells seeded in six-well plates $\left(1 \times 10^{6}\right.$ cells/well) were transfected with the appropriate plasmids using Cellfectin reagent (Invitrogen). After transfection for $16-18 \mathrm{~h}$, the cells were heat shocked in a $42{ }^{\circ} \mathrm{C}$ water bath for $30 \mathrm{~min}$ and then returned to $27^{\circ} \mathrm{C}$. At 1 or $3 \mathrm{~h}$ post-heat shock, the cells were washed with PBS and lysed with SDS sample buffer. Proteins were separated by Tricine-SDS/12.5\% polyacryla- mide gel electrophoresis (PAGE) and transferred to Immobilon-P membranes (Millipore). V5-tagged fusion proteins were detected with rabbit anti-V5 antibody (1:10000; Sigma) and goat anti-rabbit lgG-horseradish peroxidase (HRP) conjugate (1:10000; Sigma). Rpr-FLAG protein was detected with mouse anti-FLAG antibody (1:5000; Sigma) and goat anti-mouse IgG-HRP conjugate (1:10000; Sigma).

\subsection{Co-immunoprecipitation}

SF-9 cells were transfected and subjected to heat shock as described above. At $1 \mathrm{~h}$ post-heat shock, the cells were washed with PBS and lysed in $100 \mu \mathrm{l}$ of NP-40 lysis buffer ( $50 \mathrm{mM}$ Tris- $\mathrm{HCl}, \mathrm{pH} 8.0,150 \mathrm{mM} \mathrm{NaCl}, 1.0 \%$ Nonidet $\mathrm{P}-40$ ) supplemented with a protease inhibitor tablet (Roche). The lysis procedure was carried out on ice for $10 \mathrm{~min}$ with occasional shaking. The lysate was centrifuged at $12000 \mathrm{~g}$ for $5 \mathrm{~min}$ and an aliquot of the supernatant $(10 \mu \mathrm{l})$ was reserved for immunoblot analysis of the total cell lysate to confirm the expression of the transfected genes. An adequate amount of supernatant was then incubated with $10 \mu \mathrm{l}$ of anti-FLAG M2 affinity gel (Sigma) or incubated with $1 \mu \mathrm{l}$ of rabbit anti-V5 antibody (Sigma) and $20 \mu \mathrm{l}$ of protein $\mathrm{A}$ 
agarose (Sigma) at $4^{\circ} \mathrm{C}$ overnight with rotation. The gel or agarose was then washed five times in $150 \mu \mathrm{l}$ of NP-40 lysis buffer. Aliquots of the total cell lysates and immunoprecipitates were separated by Tricine-SDS/12.5\% PAGE and transferred to Immobilon-P membranes (Millipore). V5-tagged fusion proteins were detected with rabbit antiV5 antibody (Sigma) and goat anti-rabbit IgG-HRP conjugate (Sigma). Rpr-FLAG protein was detected with mouse M2 anti-FLAG monoclonal antibody (Sigma) and goat anti-mouse IgG-HRP conjugate (Sigma).

\subsection{In vitro binding assay}

To prepare the cell lysates containing Rpr-FLAG, Rpr-FLAG and P35 expression constructs were cotransfected into SF-9 cells. To prepare the lysates containing the SfIAP or various truncated PmIAP proteins, the corresponding expression constructs were transfected into SF-9 cells. At $1 \mathrm{~h}$ post-heat shock, the cell lysates were prepared as described above. The lysates containing Rpr-FLAG were mixed with the lysates containing the appropriate proteins, and incubated with anti-FLAG $\mathrm{M} 2$ affinity gel at $4{ }^{\circ} \mathrm{C}$ overnight with rotation. The gels were then washed five times with lysis buffer, and the immunoprecipitates were analyzed by immunoblotting as described above.

\subsection{Immunofluorescence microscopy}

SF-9 cells seeded on cover glasses $\left(10^{5}\right.$ cells per well in 4-well plates) were transfected with appropriate plasmids using Cellfectin reagent (Invitrogen) and heat shocked as described above. At $1 \mathrm{~h}$ after heat shock, cells were washed twice with PBS, fixed with $4 \%$ paraformaldehyde in PBS for $10 \mathrm{~min}$ at $4{ }^{\circ} \mathrm{C}$, treated with $0.1 \%$ Triton $\mathrm{X}-100$ in $4 \%$ paraformaldehyde/PBS for $3 \mathrm{~min}$ at $4{ }^{\circ} \mathrm{C}$, and then washed twice with PBS. After blocking with PBS containing $5 \%$ bovine serum albumin and $2 \%$ normal goat serum for $16 \mathrm{~h}$ at $4{ }^{\circ} \mathrm{C}$, the cells were treated with a polyclonal rabbit anti-V5 antibody (1:3000 in PBS; Sigma) or with a monoclonal mouse anti-FLAG M2 antibody (1:1000 in PBS; Sigma) or with both ( $3 \mathrm{~h}$ at room temperature). The cells were then washed 3 times (10 min each time) with PBST (0.2\% Tween 20 in PBS) and reacted either with carboxymethylindocyanine (Сy3) dye-conjugated goat anti-rabbit IgG antibody (1:1000 in PBS; Sigma) or with fluorescein isothiocyanate (FITC)conjugated goat anti-mouse IgG antibody (1:300 in PBS; Sigma), or else reacted with both for $2 \mathrm{~h}$ at room temperature. Counterstaining of the nucleus was performed with 4'-6'-diamidino-2-phenylindole dihydrochloride (DAPI) (Vector Laboratories). After washing three times $(10 \mathrm{~min}$ each time) with PBST, the cover glasses were wet mounted with anti-fade mounting media (Fluka). During all of the above procedures, the cells were kept in darkness. Fluorescence signals were examined using a fluorescence microscope (Olympus IX70).

\subsection{Statistical methods}

All quantitative data are presented as mean \pm S.D. $(n=3)$. Significant differences between pairs of groups were determined using Student's two-tailed $t$-test. A $P$-value $<0.05$ was considered statistically significant.

\section{Results}

\subsection{Isolation and sequence analysis of the PmIAP CDNA}

Complete sequencing of the PmTwI09F1 cDNA clone revealed a cDNA fragment of 2222 bp that encoded the last 60 amino acids of PmIAP, while most (2039 bp) of the rest of the sequence corresponded to the $3^{\prime}$ untranslated region (UTR). After $5^{\prime}$ RACE, the full-length $4769 \mathrm{bp}$ transcript of PmIAP was obtained and its complete sequence was deposited in GenBank under accession no. EF114675. The full-length PmIAP transcript contains a 5'UTR of $633 \mathrm{bp}$, an open reading frame (ORF) of $2097 \mathrm{bp}$, and a 3'UTR of $2039 \mathrm{bp}$. The 3'UTR contains a consensus polyadenylation signal (AATAAA) $11 \mathrm{bp}$ upstream from the poly $(A)$ tail. The ORF encodes a protein consisting of 698 amino acid residues with a calculated molecular mass of $76.8 \mathrm{kDa}$. The AUG start codon of this ORF is located within a favorable context for translation [24] and it is preceded by upstream stop codons in all three reading frames. ScanProsite analysis revealed that the predicted PmIAP protein contains three BIR domains, followed by a RING domain at its $C$ terminus. This is structurally similar to Drosophila IAP2 (DIAP2) as well as to human X-chromosome-linked IAP (XIAP), cIAP1 (cellular inhibitor of apoptosis-1), and CIAP2 (Fig. 1).

The deduced amino acid sequences of the PmIAP BIR and RING domains were aligned with the corresponding domains of other IAP family members, including Spodoptera frugiperda IAP (SfIAP), Drosophila IAP1 and IAP2 (DIAP1 and DIAP2), and human XIAP (Fig. 2). The BIR2 and BIR 3 domains of PmIAP both have the conserved BIR motif of $\mathrm{CX}_{2} \mathrm{CX}_{6} \mathrm{WX}_{3} \mathrm{DX}_{5} \mathrm{HX}_{6} \mathrm{C}$ [8]. However, in the BIR1 domain, the conserved $\mathrm{W}$ residue has been replaced with $\mathrm{K} 59$ (see the asterisk in Fig. 2) and two extra amino acids have been inserted in front of the conserved D65 residue. The alignments showed that BIR1 is the least conserved of the three BIR domains, while BIR3 and RING are the most conserved.

\subsection{Tissue distribution of PmIAP mRNA}

To investigate the tissue distribution of PmIAP transcripts, total RNAs were extracted from various $P$. monodon tissues and subjected to RT-PCR analysis. Fig. 3 shows that the PmIAP transcript was expressed in all the analyzed tissues, including epithelium, gill, heart, hepatopancreas, hemocyte, intestine, lymphoid organ, nerve, and stomach.

\subsection{Neither PmIAP nor SfIAP was able to block actinomycin D-induced apoptosis in Sf-9 cells}

In this study, to characterize the anti-apoptotic activities of PmIAP, three other anti-apoptosis proteins, SfIAP, P35, and WSSV449, were included for comparison. ActD induces apoptosis in SF cells [7], but this apoptotic response can be blocked by P35, as well as by some baculoviral and insect 


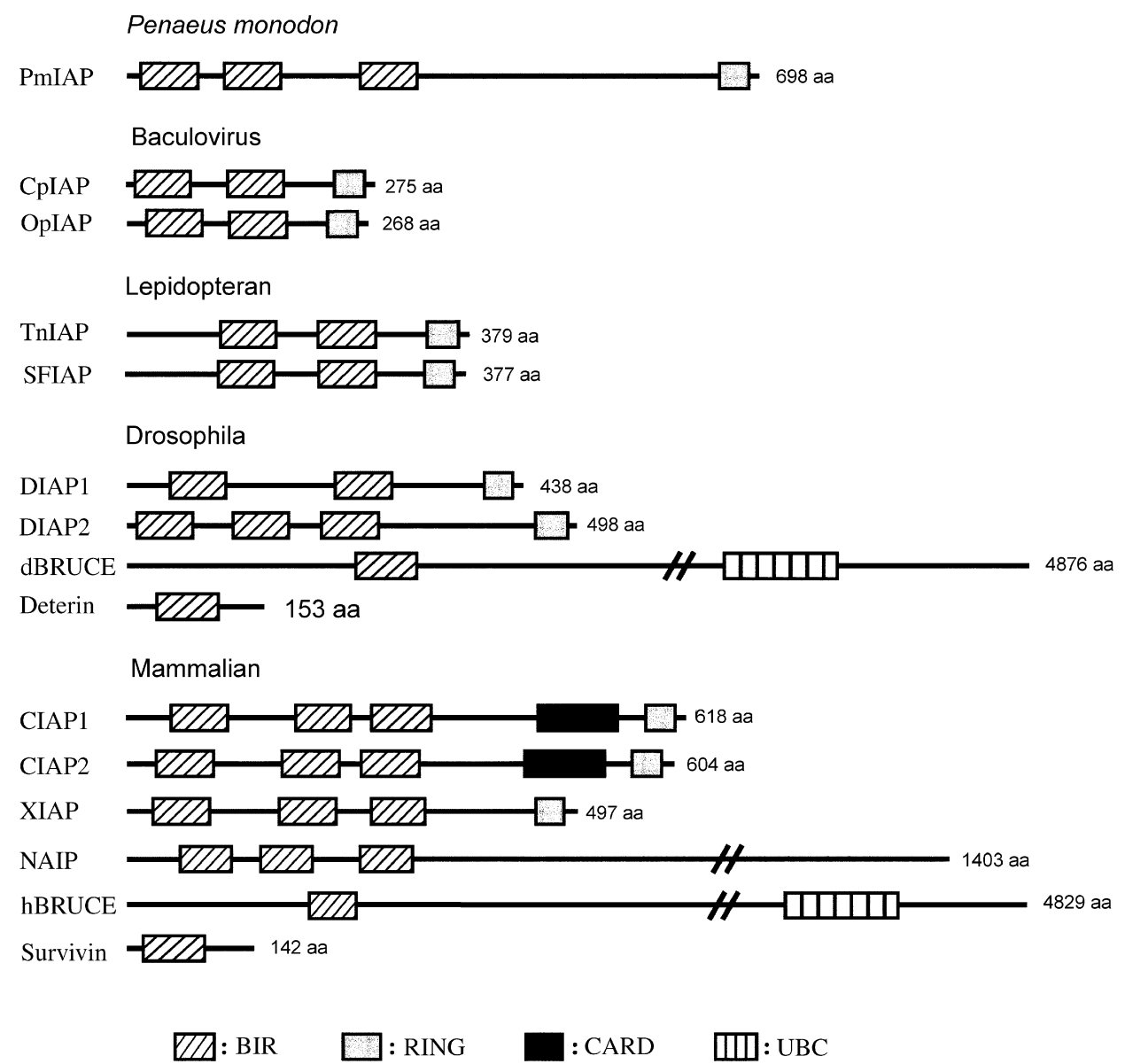

Fig. 1 Schematic representation of the structural motifs of PmIAP and several other IAP proteins. The approximate positions of the BIR, RING, CARD, and UBC domains are shown, and the total amino acid length is indicated. The possession of a RING domain in an IAP confers E3 ubiquitin protein ligase activity, while the possession of a ubiquitin-conjugating domain (UBC) is predicted to confer E2 activity. CARD refers to caspase recruitment domain and its function in CIAP1 and CIAP2 remains unknown. This figure is modified from Hay [43].

BIR1

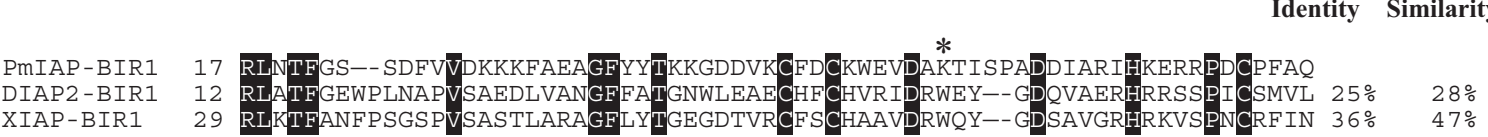

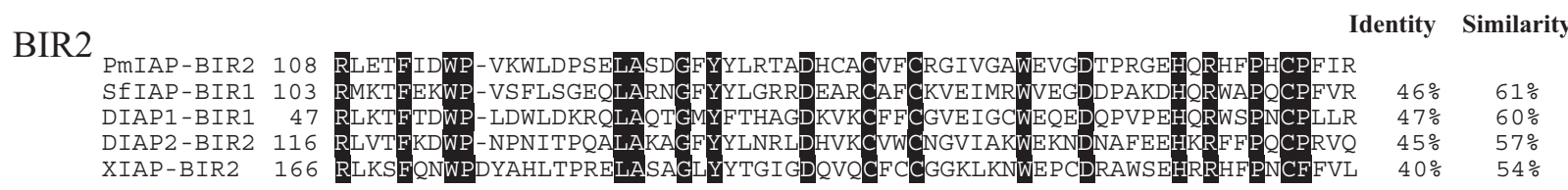

BIR3
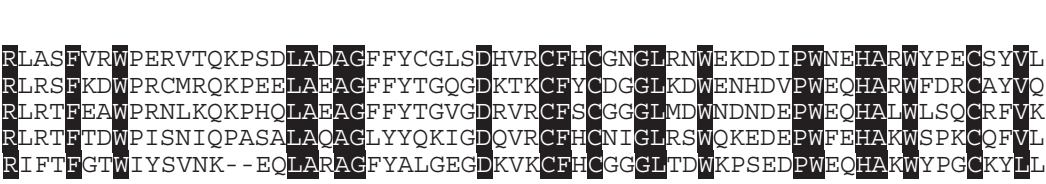

Identity Similarity

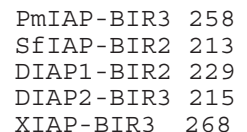

RING

Identity Similarity

$\begin{array}{llllll}\text { PmIAP-RING } & 651 & \text { CKVCMDAEMDVVFLPCAHMVTCASCAVALTQCPICR } & & \\ \text { SEIAP-RING } & 330 & \text { CKICYAEERNVCFVPCGHVVACAKCALAADKCPMCR } & 52 \% & 72 \% \\ \text { DIAP1-RING } & 391 & \text { CKICYGAEYNTAFLPCGHVACAKCASSVTKCPLCR } & 55 \% & 75 \% \\ \text { DIAP2-RING } & 451 & \text { CKVCLDEEVGVVFLPCGHLATCNOCAPSVANCPMCR } & 58 \% & 75 \% \\ \text { XIAP-RING } & 450 & \text { CKICMDRNIAIVEVPCGHLVTCKQCAEAVDKCPMC } & 52 \% & 75 \%\end{array}$

Fig. 2 Multiple sequence alignments of the BIR1, BIR2, BIR3, and RING domains of PmIAP with the corresponding domains of other IAP family members. Identical residues are indicated in black. The amino acid sequence identities and similarities in each aligned domain are also indicated. The asterisk in the PmIAP BIR1 domain indicates the K59 residue that replaces the invariant W. GenBank accession numbers for SfIAP, DIAP1, DIAP2, and XIAP are AX213188, Q24306, Q24307, and P98170, respectively. 
IAPs $[22,25,26]$. Here, we determined whether PmIAP could also block ActD-induced apoptosis in SF-9 cells. First we confirmed that all constructs successfully expressed the indicated V5-tagged proteins (Fig. 4A). Expression levels were similar, except for SfIAP, which was slightly lower (Fig. 4A, lane 3). For the P35, in addition to the major protein band, a number of higher molecular weight products were also detected (Fig. 4A, lane 4). We interpret these bands to be protein complexes formed from P35 and some cellular proteins, because similar P35-associated high molecular weight complexes were also reported when P35 was transiently expressed in mammalian cells [27]. We also note that the apparent molecular mass of PmIAP is about $110 \mathrm{kDa}$ (Fig. 4A, lane 2), which is larger than its calculated molecular mass of about $77 \mathrm{kDa}$. Fig. $4 \mathrm{~B}$ shows that P35 and,

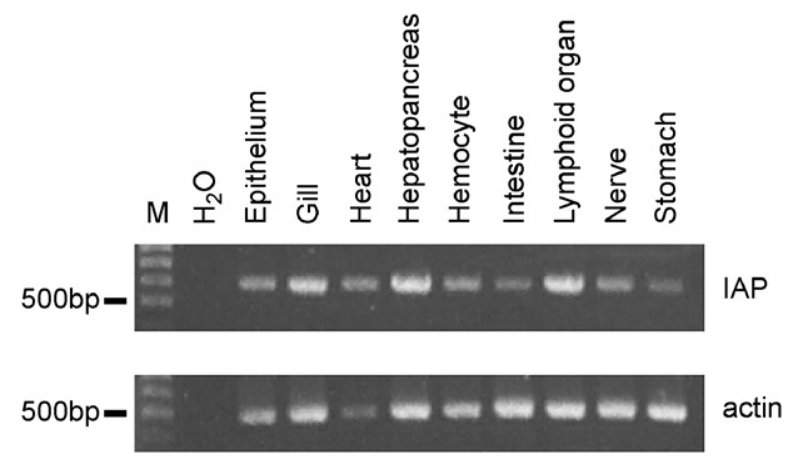

Fig. 3 Tissue distribution of PmIAP mRNA by RT-PCR analysis. $\mathrm{H}_{2} \mathrm{O}$ is included as negative control. $M$ indicates molecular weight marker (100 bp ladder). to a lesser extent, WSSV449 could significantly prevent the cells from undergoing ActD-induced apoptosis (both $P<0.05)$, while neither PmIAP nor SfIAP demonstrated any anti-apoptotic effect.

\subsection{PmIAP inhibits Rpr-induced apoptosis in SF-9 cells}

In SF-9 cells, both baculoviral and Drosophila IAPs have been shown to block the apoptosis induced by $\operatorname{Rpr}[28,29]$. Therefore, in the present study, we investigated the ability of PmIAP to inhibit Rpr-induced apoptosis in SF-9 cells. Fig. 4C shows that PmIAP, SfIAP, P35, and WSSV449 all significantly blocked Rpr-induced apoptosis (all $P<0.05$ ). However, while the blocking ability of SfIAP and P35 was quite strong, PmIAP was the least effective of these four proteins.

\subsection{PmIAP interacts with Rpr and delays its degradation in insect cells}

The physical interactions of baculoviral and Drosophila IAPs with Rpr not only inhibited its apoptotic activity but also prevented its degradation [28]. To investigate whether PmIAP and SfIAP could also interact with Rpr and prevent its degradation, we generated a construct that expressed an Rpr protein fused with a C-terminal FLAG-His6 tag (Rpr-FLAG). A time course study was then conducted to investigate the influence of these anti-apoptosis proteins on the stability of Rpr-FLAG. The results showed that, at $1 \mathrm{~h}$ post-heat shock, Rpr-FLAG proteins were detected in cells

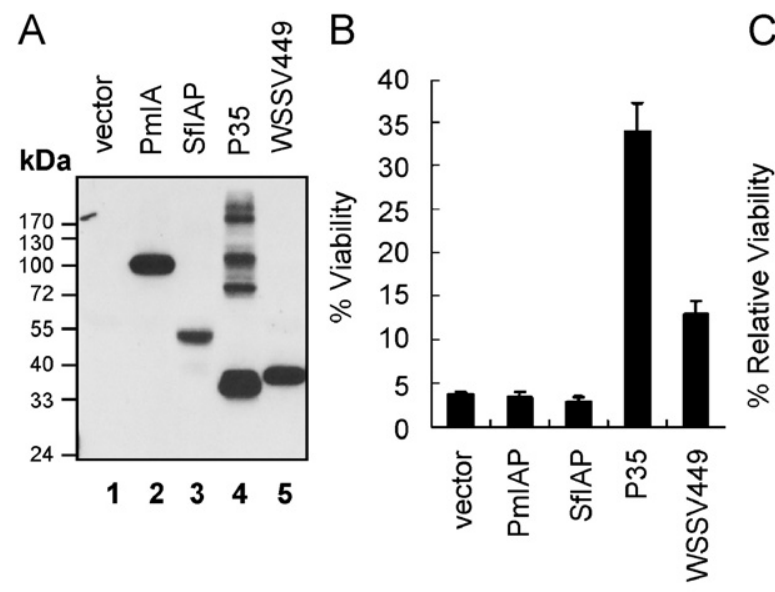

C

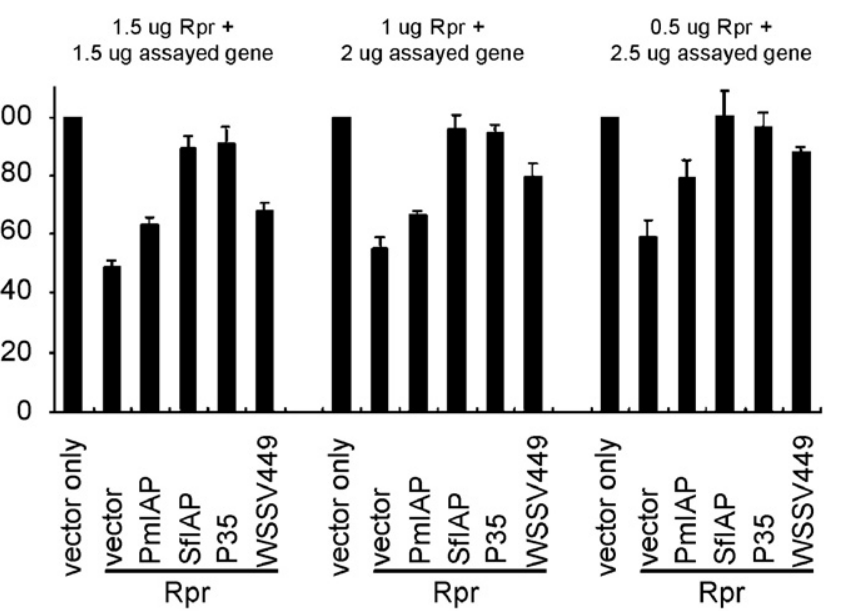

Fig. 4 In SF-9 cells, PmIAP inhibits apoptosis induced by Drosophila Rpr protein, but cannot block apoptosis induced by ActD. (A) The expression of PmIAP, SfIAP, P35, and WSSV449 in SF-9 cells was confirmed by immunoblot analysis with rabbit anti-V5 antibody. SF-9 cells transfected with an empty expression plasmid pDHsp/V5-His were included as negative control (vector). (B) SF-9 cells were transfected with either empty expression plasmid (vector) or the plasmids expressing PmIAP, SfIAP, P35, or WSSV449. At $4 \mathrm{~h}$ after heat shock, ActD was added and, 14-16 h later, cell viability was measured. Data represent the means of three replicates. Standard deviations are indicated by error bars. The differences between vector and P35, between vector and WSSV449, and between P35 and WSSV449 are statistically significant ( $t$-test; all $P<0.05)$. (C) An Rpr expression construct was cotransfected into SF-9 cells with either empty plasmid (vector) or with the plasmids expressing PmIAP, SfIAP, P35, or WSSV449. SF-9 cells transfected only with empty plasmid were included as a negative control (vector only). At $5 \mathrm{~h}$ after heat shock, the number of viable cells was determined and the relative percentage of viability was calculated. The amounts of plasmid DNAs used for transfection are indicated in the figure. The results shown are the means of three replicates. Standard deviations are indicated by error bars. At the same input DNA amounts, the differences between the vector cell group and the other cell groups are statistically significant $(t$-test; all $P<0.05)$. 

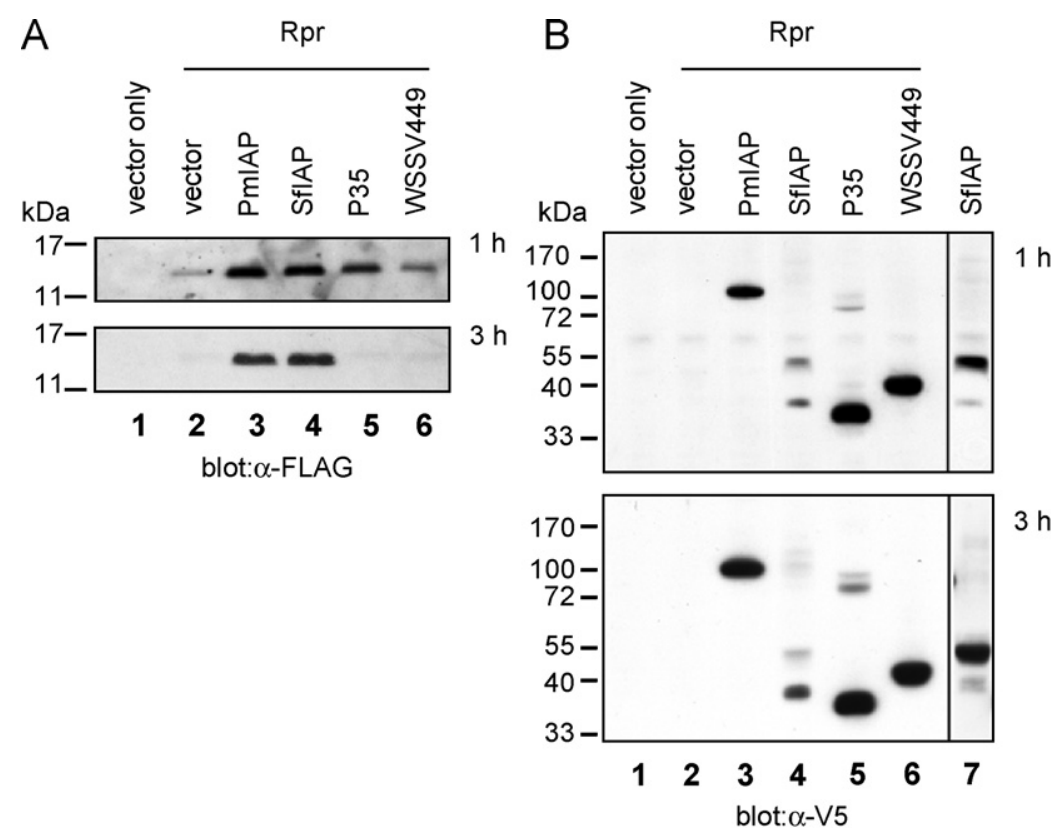

Fig. 5 PmIAP and SfIAP prevent the degradation of Rpr protein in SF-9 cells although SfIAP is relatively unstable in apoptotic cells. Rpr-FLAG expression construct $(1 \mu \mathrm{g})$ was cotransfected into SF-9 cells with either the empty plasmid (vector) or the plasmids expressing PmIAP, SfIAP, P35, or WSSV449 (3 $\mu \mathrm{g})$. SF-9 cells only transfected with empty plasmid were included as negative control (vector only). (A) At $1 \mathrm{~h}$ (upper panel) and $3 \mathrm{~h}$ (lower panel) after heat shock, the cell lysates were harvested and subjected to immunoblot analysis with anti-FLAG antibody to detect the presence of Rpr-FLAG. (B) The same lysates ( $1 \mathrm{~h}$ : upper panel; and $3 \mathrm{~h}$ : lower panel) were also analyzed with immunoblotting using anti-V5 antibody to detect PmIAP, SfIAP, P35 and SSV449. For comparison, the lysates from cells transfected with SfIAP only were also analyzed (lane 7).

that coexpressed PmIAP, SfIAP, P35, or WSSV449 (Fig. 5A, upper panel, lanes 3-6), while in cells expressing Rpr-FLAG alone the Rpr-FLAG protein level was much lower (Fig. 5A, upper panel, lane 2 ). At $3 \mathrm{~h}$ post-heat shock, Rpr-FLAG was detected only in cells that coexpressed PmIAP and SfIAP (Fig. 5A, lower panel, lanes 3 and 4). Fig. 5B shows the expression levels of the four anti-apoptosis proteins at 1 and $3 \mathrm{~h}$ post-heat shock. The amounts of PmIAP, P35, and WSSV449 increased during this time interval (Fig. 5B, lanes 3-6), while SfIAP appeared to be quite unstable in Rpr-FLAGcoexpressing cells. In the absence of Rpr-FLAG, SfIAP protein levels increased over time (Fig. 5B, lane 7), whereas in the Rpr-FLAG-coexpressing cells, at $3 \mathrm{~h}$ post-heat shock, most of the expressed SfIAP protein was truncated (Fig. 5B). We hypothesize that the truncated SfIAP was generated through cleavage of the full-length SfIAP by the activated caspase in the Rpr-induced apoptotic cells. This explanation is based on the fact that Drosophila DIAP1, which is structurally similar to SfIAP (Fig. 1), is degraded by caspases in apoptotic cells in response to Rpr expression [30,31], and we note that the predicted caspase-cleavage sites in SfIAP correspond to those that have been identified in DIAP1.

Next, we determined whether antibody to the Rpr-FLAG protein could co-immunoprecipitate these anti-apoptosis proteins. The result (Fig. 6A) showed that, although RprFLAG proteins were efficiently precipitated by the anti-FLAG antibody in all four samples, PmIAP was the only protein to co-precipitate with Rpr-FLAG (upper panel, lane 1), even though both P35 and WSSV449 (if not SfIAP) were being efficiently expressed (see Fig. 5B, lanes 4, 5, and 6). A reciprocal experiment that used anti-V5 antibody to precipitate the complexes also showed that only V5-tagged PmIAP could specifically co-precipitate the FLAG-tagged Rpr (data not shown). In both of these experiments, the apparent inability of SfIAP to co-precipitate with Rpr-FLAG was quite surprising. Possible explanations might be the relatively low expression levels of SfIAP (compare with PmIAP, P35, and WSSV449 in Figs. 4A and 5B) and the instability of SfIAP in the presence of Rpr (Fig. 5B, lanes 4 and 7), so that although the Rpr-FLAG protein was efficiently precipitated by the anti-FLAG antibody, the amount of coprecipitated SfIAP may have been too low to be detected. To resolve this, an in vitro binding assay was conducted. The lysates from cells coexpressing Rpr-FLAG and P35 were mixed with the lysates from cells that expressed SfIAP, and then incubated with anti-FLAG antibody resin to immunoprecipitate the Rpr-FLAG proteins. Immunoblot analysis of the immunoprecipitates with anti-V5 antibody showed that SfIAP co-precipitated with Rpr-FLAG (Fig. 6B). A negative control without the addition of Rpr-FLAG showed the specificity of the in vitro binding assay.

\subsection{PmIAP and Rpr share the same subcellular locations}

To investigate whether PmIAP and Rpr are co-localized in the overexpressing cells, PmIAP was expressed in SF-9 cells with or without Rpr-FLAG and then assayed with indirect immunofluorescence microscopy. SfIAP, P35, and WSSV449 were also included for comparison. Immunofluorescent signals for Rpr-FLAG were detected in cells that coexpressed 
A
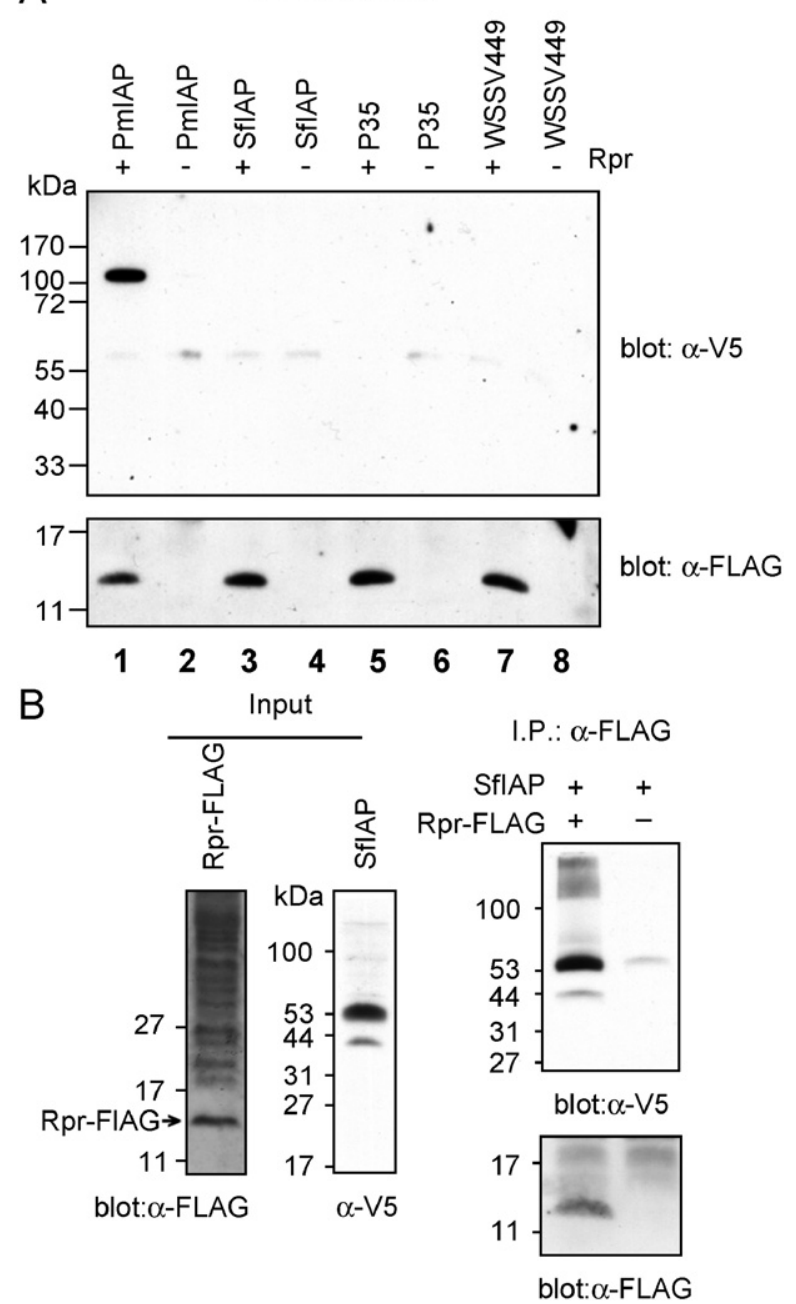

Fig. 6 PmIAP and SfIAP interact with Rpr protein in SF-9 cells. (A) The Rpr-FLAG expression construct was cotransfected into SF-9 cells with the plasmids expressing V5-tagged PmIAP, SfIAP, P35 or WSSV449. At $1 \mathrm{~h}$ after heat shock, the cell lysates were harvested and immunoprecipitated with anti-FLAG M2 affinity resin. The immunoprecipitated complexes were then subjected to immunoblot analysis probing with anti-V5 or anti-FLAG antibodies. Cell lysates prepared from cells that only expressed PmIAP, SfIAP, P35, or WSSV449 (lane 2, 4, 6, and 8, respectively) were also subjected to immunoprecipitation and immunoblot to confirm the specificity of the anti-FLAG M2 affinity resin. (B) The lysates from Rpr-FLAG and P35-coexpressing cells were mixed with the lysates from SfIAP-expressing cells (input), and immunoprecipitated with anti-FLAG M2 affinity resin. The immunoprecipitated complexes were then subjected to immunoblot analysis probing with anti-V5 or anti-FLAG antibodies.

PmIAP, SfIAP, P35, or WSSV449 (Fig. 7, column 3), whereas in cells expressing only Rpr-FLAG, no immunofluorescent signal was detected (data not shown). This suggested that the amount of Rpr-FLAG protein in cells that coexpressed PmIAP, SfIAP, P35, or WSSV449 was higher than in cells expressing only Rpr-FLAG, which is consistent with our immunoblot result (Fig. 5A). Column 3 of Fig. 7 suggests that Rpr-FLAG was generally diffused in the cytoplasm, while some cells also showed punctate signals. Signal intensity, the number of cells showing punctate signals, and the number of punctates themselves were highest when Rpr-FLAG was coexpressed with PmIAP. Row 1 of Fig. 7 shows that the staining pattern for PmIAP was also changed in the presence of Rpr-FLAG (cf cols 1 and 2). The strong punctate staining of PmIAP (row 1 , col 2) almost exactly matches that of Rpr-FLAG (row 1, col 3), which suggests that both PmIAP and Rpr-FLAG were co-localized to the same subcellular locations. Conversely, the staining patterns of SfIAP, P35, and WSSV449 were mostly unaffected by the presence of Rpr (Fig. 7, cf cols 1 and 2).

\subsection{BIR2 and BIR3 are the minimal regions for PmIAP to interact with Rpr and prevent its pro- apoptotic activity}

To determine which regions of PmIAP are important for blocking Rpr-induced apoptosis and for binding to Rpr, a series of PmIAP truncation constructs were prepared (Fig. 8A). Rpr and the truncated PmIAPs were coexpressed in SF-9 cells, and the relative viability of the transfected cells was determined. All the constructs except RING and BIR1 were able to block apoptosis with an efficiency that was seemingly related to the number of BIR domains, and this blocking effect was statistically significant for the PmIAP, BIR, BIR1+2, and BIR2+3 constructs (Fig. 8B). BIR, which was a truncated PmIAP construct with three BIR domains and no RING domain, showed the highest inhibitory activity. Since this was even higher than that of the full-length PmIAP, it may be that the RING domain negatively regulates PmIAP's blocking of Rpr-induced apoptosis. Constructs with two BIR domains (BIR1+2 and BIR2+3) had a lower inhibitory activity, while constructs with a single BIR domain (BIR2 and BIR3) had the lowest inhibitory activity of all. Although both BIR2 and BIR3 showed no statistically significant effect on Rpr, the relative viability of the corresponding transfected cells was always higher than that of the control (vector) cells. Interestingly, both the BIR1 and RING constructs actually enhanced the apoptosis induced by Rpr. This effect was statistically significant only for BIR1, but the relative viability of the RING-transfected cells was also constantly lower than that of the control cells. We note, however, that when BIR1 or RING was expressed in SF-9 cells in the absence of Rpr, no cell death was observed (data not shown).

The PmIAP truncation constructs were further tested for their ability to delay the degradation of Rpr-FLAG (Fig. 8C). At $1 \mathrm{~h}$ post-heat shock, Rpr-FLAG was detected in all of the cotransfected cells. The cells that coexpressed PmIAP, BIR, $\mathrm{BIR} 3, \mathrm{BIR} 1+2$, or BIR2+3 had high amounts of Rpr-FLAG, while the low expression levels in the RING, BIR1, and BIR2coexpressing cells were similar to the expression levels in the cells expressing only Rpr-FLAG. At $3 \mathrm{~h}$ post-heat shock, the BIR2-coexpressing cells still had a low level of Rpr-FLAG, but no Rpr-FLAG was detected in cells coexpressing BIR1 or RING, or in cells expressing only Rpr-FLAG. Meanwhile, high amounts of Rpr-FLAG were still being detected in the PmIAP, BIR, BIR3, BIR1+2, and BIR2+3-coexpressing cells. The expression of each PmIAP truncation construct was also confirmed using anti-V5 antibody (Fig. 8C, lower panel).

In vitro binding assays were then performed to determine which of these truncated PmIAP proteins interact with RprFLAG. Immunoblot analysis of the immunoprecipitates with 


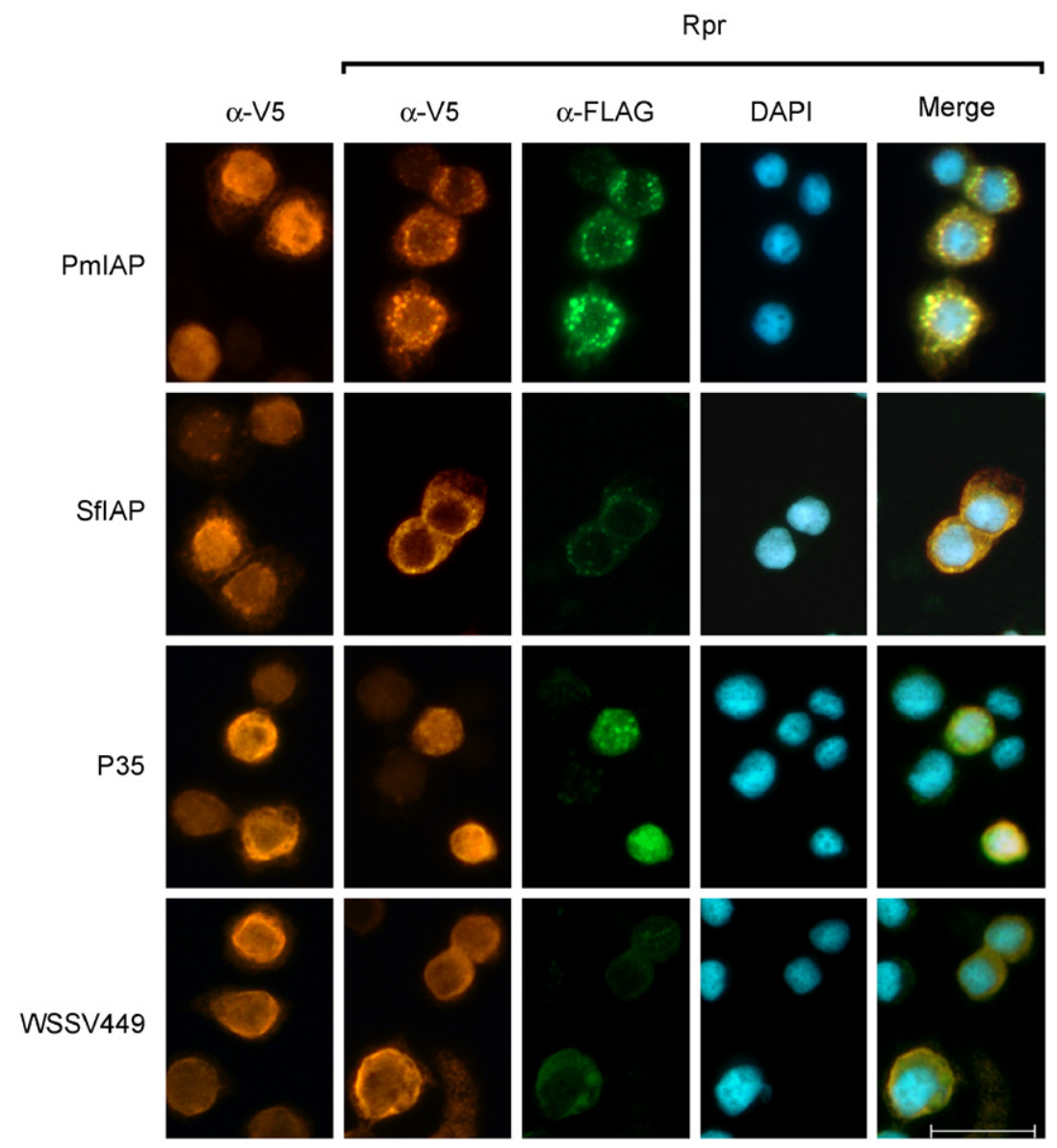

Fig. 7 Co-localization of PmIAP and Rpr-FLAG. Expression plasmids encoding PmIAP (row 1), SfIAP (row 2), P35 (row 3 ), or WSSV449 (row 4) were cotransfected into SF-9 cells either with (cols 2-5) or without (col 1) the plasmid expressing Rpr-FLAG. At $1 \mathrm{~h}$ after heat shock, the cells were processed and analyzed by indirect immunofluorescence. PmIAP, SfIAP, P35, and WSSV449 were visualized using rabbit anti-V5 antibody and Cy3-conjugated goat anti-rabbit IgG antibody (columns 1 and 2). Rpr-FLAG was visualized with monoclonal mouse anti-FLAG M2 antibody and FITC-conjugated goat anti-mouse IgG antibody (column 3). Nuclei were visualized by counterstaining with DAPI (column 4). Column 5 shows the merged Cy3, FITC, and DAPI signals. Scale bar equals $25 \mu \mathrm{m}$.

anti-V5 antibody showed that, except for RING and BIR1, all the other truncated proteins could co-precipitate with RprFLAG (Fig. 9). This suggests that the PmIAP BIR2 and BIR3 domains are the minimal regions that bind to Rpr (Fig. 9A). In Fig. 9, the amounts of the co-precipitated BIR2, BIR3, and BIR1+2 are significantly lower than those of PmIAP, BIR, and $\mathrm{BIR2}+3$. This is probably because BIR2, BIR3, and BIR1+2 each have only one Rpr-binding site, whereas the other three proteins each have two Rpr-binding sites. The results also showed that, among the three truncated proteins with a single Rpr-binding site, the amount of precipitated BIR1+2 was higher than that of BIR2 or BIR3 (compare the coimmunoprecipitated bands with the respective IgG bands in Fig. 9A and $B$, noting that the photographic exposure time was $\sim 6 \times$ greater for the I.P. panel in Fig. 9A). This suggests that although BIR1 does not bind to Rpr itself, it nevertheless enhances the binding of BIR1+2 to Rpr.

\section{Discussion}

In this paper, we report the first cloning and characterization of a novel IAP family member, PmIAP, from the tiger shrimp, $P$. monodon. PmIAP consists of 698 amino acids and has three BIR domains followed by a RING domain at the $C$ terminus (see Fig. 1). We demonstrated that PmIAP was able to inhibit apoptosis in SF-9 cells induced by Rpr, but not the apoptosis induced by ActD (Fig. 4B,C). Further study revealed that PmIAP and $\mathrm{Rpr}$ physically interacted with each other (Fig. 6A and 9), and that they both shared the same subcellular locations (Fig. 7). Truncation analysis showed that the PmIAP BIR2 and BIR3 domains played important roles in inhibiting Rpr-induced apoptosis in SF cells, and that the BIR1 domain increased this inhibitory ability even though BIR1 did not bind to Rpr directly (Figs. 8 and 9). Since ectopic expression of Rpr induces apoptosis in vertebrate cells as well as in invertebrate cells [29,32,33], the results presented here imply that PmIAP exerts its antiRpr activity by means of mechanisms that are evolutionarily conserved across crustaceans, insects, and mammals.

In this study, three other anti-apoptosis proteins were included for comparison. SfIAP is an endogenous IAP of S. frugiperda cells, while P35 is a well-studied, baculoviral inhibitor of many caspases [34-36]. By contrast, there have been no further studies of WSSV449 (ORF390), since it was first identified [21]. Wang et al. [21] showed that when 

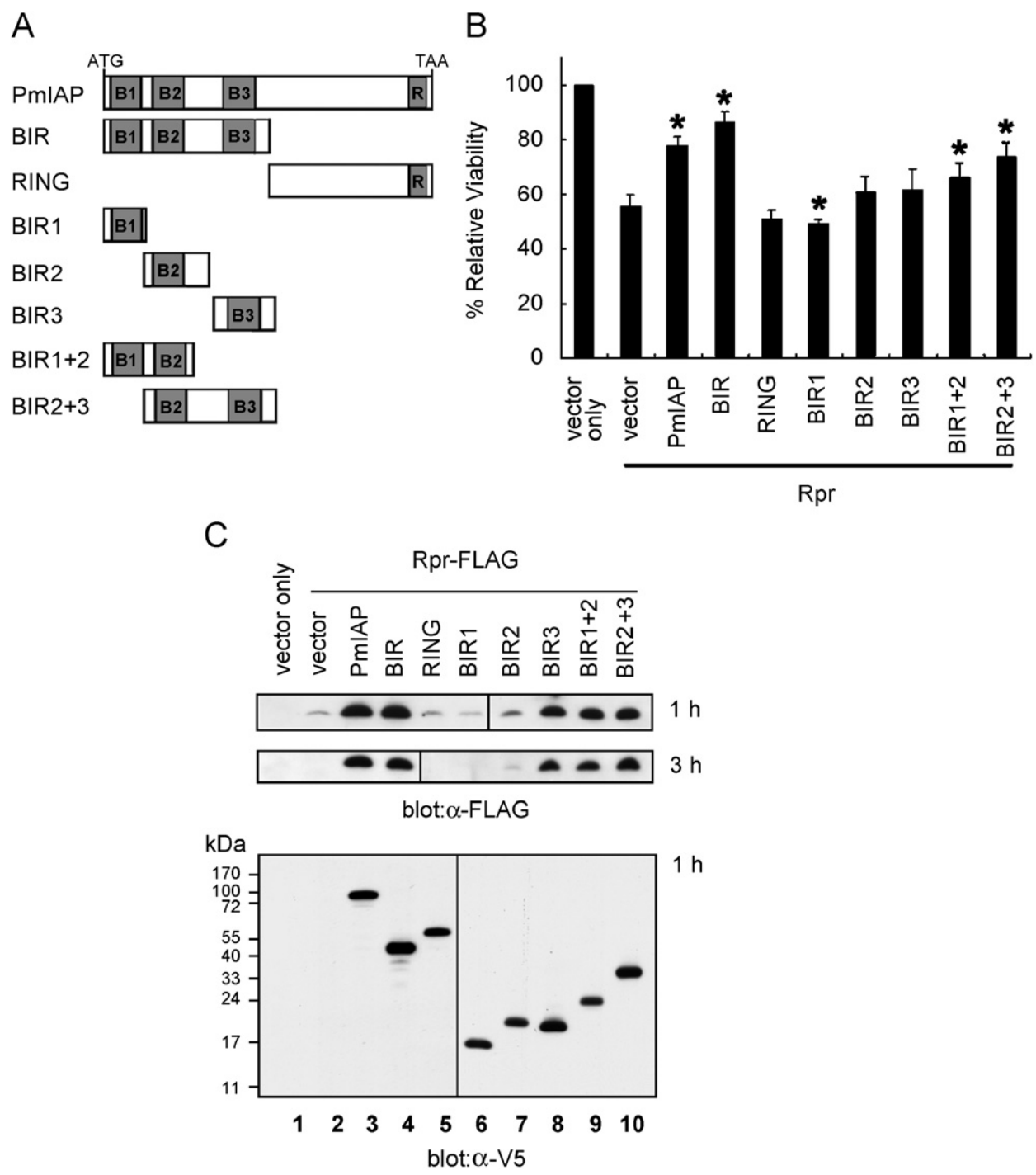

Fig. 8 Mapping the region[s] important for PmIAP to block Rpr-induced apoptosis in SF-9 cells. (A) Schematic diagram showing the various PmIAP truncation constructs. (B) SF-9 cells were cotransfected either with $0.5 \mu \mathrm{g}$ Rpr and $2.5 \mu \mathrm{g}$ of empty plasmid (vector) or PmIAP constructs, or transfected with empty plasmid (vector only). At $5 \mathrm{~h}$ after heat shock, the number of viable cells was determined and the relative viability was calculated. The asterisk indicates a statistically significant difference between the vector and various IAP constructs ( $t$-test; $P<0.05$ ). (C) SF-9 cells were cotransfected with Rpr-FLAG and various PmIAP constructs. At 1 and $3 \mathrm{~h}$ post-heat shock, cell lysates were harvested and subjected to immunoblot analysis probing with antibodies against anti-FLAG (upper panels) or anti-V5 (lower panel; lysates from $1 \mathrm{~h}$ post-heat shock).

apoptosis was induced in SF-9 cells by infection with a p35deficient AcMNPV, the expression of WSSV449 blocked this apoptosis and allowed the virus to replicate. From this, the authors concluded that WSSV449 was functionally similar to P35. Here, we have shown that, in SF-9 cells, WSSV449 blocks apoptosis when induced either by ActD or by Rpr (Fig. 4B, C). This suggests that WSSV449 functions through a conserved mechanism to block apoptosis induced by different stimuli. We have also found that, in SF-9 cells, WSSV449 directly binds to the $P$. monodon effector caspase (accession no. EF114674) and inhibits its proapoptotic activity (data not shown), and we therefore confidently conclude that WSSV449 is a caspase inhibitor. Thus, the two viral caspase inhibitors (WSSV449 and P35) blocked Rpr- and ActD-induced apoptosis, whereas the two cellular IAPs (PmIAP and SfIAP) only prevented apoptosis that was induced by Rpr (Fig. 4B, C). The inability of the cellular IAPs to block the ActD-induced apoptosis suggests that the caspase-inhibiting activity of these two IAPs is less effective than that of the two viral caspase inhibitors. We also note that, under natural conditions, shrimp cells would be the normal functional environment for the PmIAP and WSSV449 anti-apoptosis proteins. Thus, ideally, a shrimp cell line would have been used for the present studies, but, unfortunately, since no crustacean cell line has yet been established, we used SF-9 insect cells instead. It is therefore reasonable that, compared with the two insect anti-apoptosis proteins, the two shrimp anti-apoptosis proteins would function less effectively in SF-9 cells.

Studies on mammalian and Drosophila IAPs with multiple BIR domains have indicated that, although the BIR domains are similar in both structure and sequence, they are 

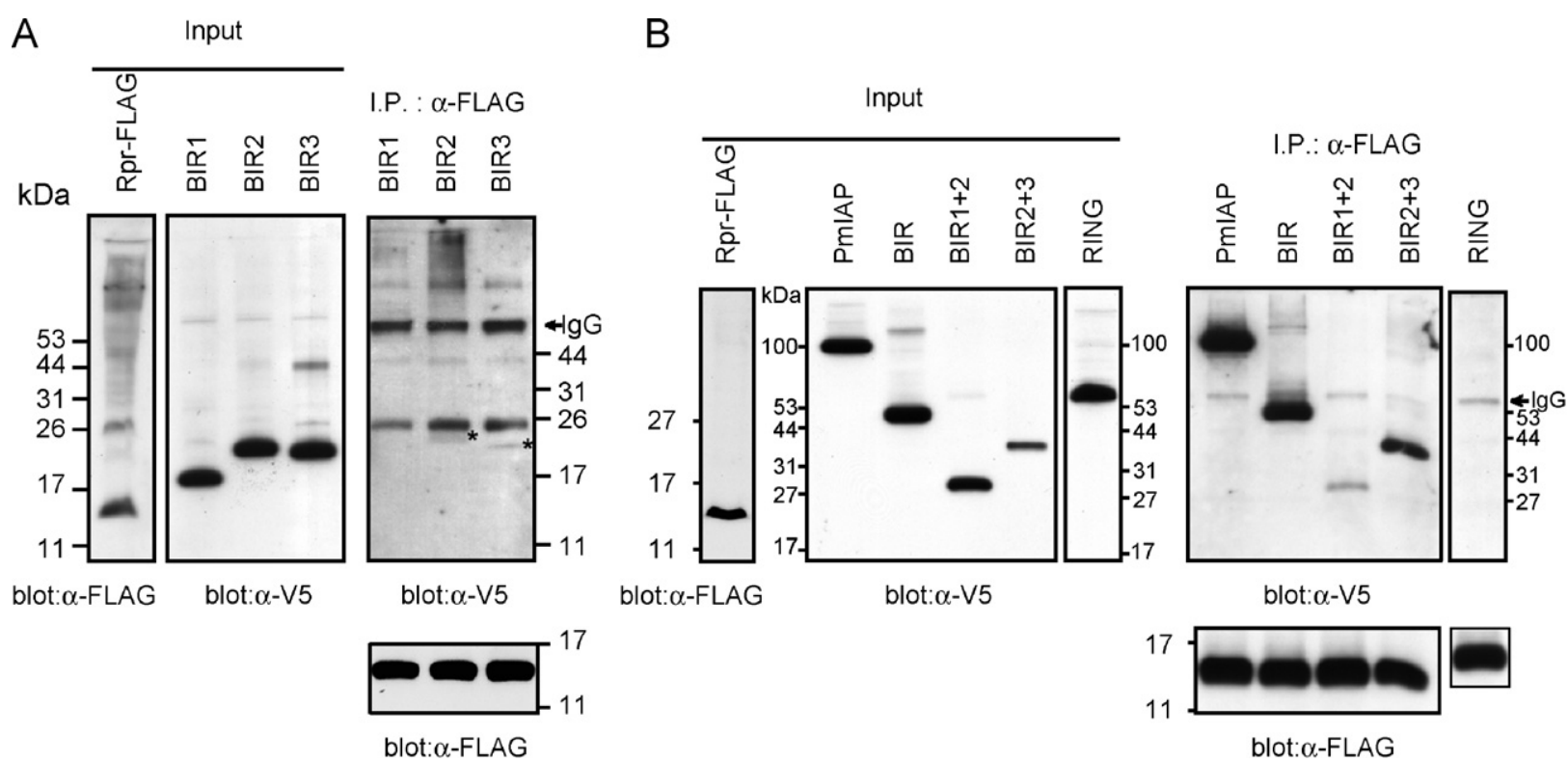

Fig. 9 In vitro binding assay to map the region[s] important for PmIAP to interact with Rpr. The lysates containing Rpr-FLAG were mixed with the lysates containing various PmIAP truncated proteins and subjected to immunoprecipitation with anti-FLAG M2 affinity resin. The immunoprecipitates were analyzed with immunoblot probing with anti-V5 or anti-FLAG antibodies. The input lysates were also analyzed with appropriate antibodies. (A) BIR1, BIR2, and BIR3 truncated proteins were assayed. Asterisks indicate the precipitated BIR2 and BIR3 proteins. (B) PmIAP and the truncated proteins BIR, BIR1+2, BIR2+3, and RING were assayed. The IgG band is indicated.

functionally and biochemically different. In mammalian XIAP, the BIR2 domain inhibits caspase-3 and -7 , and the BIR3 domain inhibits caspase-9, whereas the BIR1 domain displays no caspase-inhibiting activity and is also the least conserved of the three BIR domains [12]. Furthermore, Smac relieves the inhibition of caspases by binding to either XIAP BIR2 or BIR3, but not to BIR1, and its interaction with BIR3 is considerably stronger $[37,38]$. In the present study, the relative importance of the BIR3 domain in PmIAP is suggested by the amino acid sequence alignment (Fig. 2), which shows that the PmIAP BIR3 is highly conserved. Conversely, the BIR1 domain is the least well conserved. The results shown in Figs. 8 and 9 revealed that, among the three BIR domains, both BIR2 and BIR3 bind to Rpr and block its apoptotic activity, whereas BIR1 does not. Furthermore, BIR3 shows a slightly greater ability to delay the degradation of Rpr protein and to block the apoptosis induced by Rpr. These interactions between PmIAP and Rpr are similar to those between XIAP and Smac, suggesting that the mechanism by which an IAP interacts with an IBM protein is evolutionarily conserved across mammals and crustaceans. We note, however, that, although the PmIAP BIR1 domain showed no Rpr-binding activity, the truncated proteins that retained the BIR1 domain exhibited stronger inhibitory and binding activity to Rpr (Figs. 8 and 9, cf BIR2 vs. BIR1+2, $B I R 2+3$ vs. BIR). This suggests that BIR1 might have some regulatory role, and that PmIAP needs BIR1 to fully exert its ability to inhibit the apoptosis induced by Rpr.

Compared with Drosophila IAPs, PmIAP is structurally related to DIAP1 and DIAP2, and is most similar to DIAP2 (Fig. 1). When overexpressed, both DIAP1 and DIAP2 inhibit apoptosis [39], but, in a normal cellular context, they have quite different biological functions. DIAP1 is essential to the survival of Drosophila cells in vivo $[39,40]$ and in vitro [41], whereas although DIAP2 is required for the Imd pathway to trigger the synthesis of antimicrobial peptide, it is dispensable for Drosophila's survival [16-19]. Despite their different biological functions, both DIAP1 and DIAP2 are negatively regulated by IBM proteins through similar mechanisms. The interactions between IAPs and IBM proteins are important for functionally regulating many IAPs, and we have shown here (Figs. 6 and 7) that PmIAP also interacts with an IBM protein (Rpr). Thus, although the function[s] of PmIAP have not yet been experimentally determined, it is likely that these functions are also regulated by shrimp IBM protein[s] through direct interaction. Meanwhile, even though the biological function of PmIAP remains unknown, its importance in shrimp is suggested by its gene expression profile: PmIAP is expressed in at least two different developmental stages (postlarval and adult), and in the adult stage it is expressed ubiquitously. Until recently, very few methods were available to study in vivo gene function in shrimp, but now that RNAi technology has been successfully applied in shrimp to silence gene expression [42], the next step will be to use this technology to investigate the biological function[s] of PmIAP in shrimp.

\section{Acknowledgments}

This investigation was supported financially by the National Science Council Grants (NSC94-2317-B-002-022, NSC952317-B-002-009, and NSC95-2317-B-002-010). Both GuangHsiung Kou and Chu-Fang Lo are corresponding authors for this work. We are indebted to Paul Barlow for his helpful criticism. 


\section{References}

[1] Steller H, Grether ME. Programmed cell death in Drosophila. Neuron 1994;13:1269-74.

[2] Vaux DL, Korsmeyer SJ. Cell death in development. Cell 1990;96:245-54.

[3] Opferman JT, Korsmeyer SJ. Apoptosis in the development and maintenance of the immune system. Nat Immunol 2003; 4:410-5.

[4] Häcker G. The morphology of apoptosis. Cell Tissue Res 2000; 301:5-17.

[5] Kerr JFF, Wylie AH, Currie AR. Apoptosis: a basic biological phenomenon with wide-ranging implications in tissue kinetics. Br J Cancer 1972;26:239-57.

[6] Danial NN, Korsmeyer SJ. Cell death: critical control points. Cell 2004;116:205-19.

[7] Crook NE, Clem RJ, Miller LK. An apoptosis-inhibiting baculovirus gene with a zinc finger-like motif. J Virol 1993;67: 2168-74.

[8] Deveraux QL, Reed JC. IAP family proteins-suppressors of apoptosis. Genes Dev 1999;13:239-52.

[9] Salvesen GS, Duckett CS. IAP proteins: blocking the road to death's door. Nat Rev Mol Cell Biol 2002;3:401-10.

[10] Deveraux QL, Takahashi R, Salvesen GS, Reed JC. X-linked IAP is a direct inhibitor of cell-death proteases. Nature 1997;388: $300-4$.

[11] Roy N, Deveraux QL, Takahashi R, Salvesen GS, Reed JC. The cIAP-1 and C-IAP-2 proteins are direct inhibitors of specific caspases. EMBO J 1997;16:6914-25.

[12] Liston P, Fong WG, Korneluk RG. The inhibitors of apoptosis: there is more life than Bcl2. Oncogene 2003;22:8568-80.

[13] Ditzel M, Meier P. IAP degradation: decisive blow or altruistic sacrifice? Trends Cell Biol 2002;12:449-52.

[14] Vaux DL, Silke J. IAPs, RINGs and ubiquitylation. Nat Rev Mol Cell Biol 2005;6:287-97.

[15] Conte D, Holcik M, Lefebvre CA, LaCasse E, Picketts DJ, Wright $\mathrm{KE}$, et al. Inhibitor of apoptosis protein CIAP2 is essential for lipopolysaccharide-induced macrophage survival. Mol Cell Biol 2006;26:699-708.

[16] Kleino A, Valanne S, Ulvila J, Kallio J, Myllymaki H, Enwald H, et al. Inhibitor of apoptosis 2 and TAK1-binding protein are components of the Drosophila Imd pathway. EMBO J 2005;24:3423-34

[17] Gesellchen V, Kuttenkeuler D, Steckel M, Pelte N, Boutros M. An RNA interference screen identifies inhibitor of apoptosis protein 2 as a regulator of innate immune signalling in Drosophila. EMBO Rep 2005;6:979-84.

[18] Leulier F, Lhocine N, Lemaitre B, Meier P. The Drosophila inhibitor of apoptosis protein DIAP2 functions in innate immunity and is essential to resist Gram-negative bacterial infection. Mol Cell Biol 2006;26:7821-31.

[19] Huh JR, Foe I, Muro I, Chen CH, Seol JH, Yoo SJ, et al. The Drosophila inhibitor of apoptosis (IAP) DIAP2 is dispensable for cell survival, required for the innate immune response to Gram-negative bacterial infection, and can be negatively regulated by the reaper/hid/grim family of IAP-binding apoptosis inducers. J Biol Chem 2007;282:2056-68.

[20] Royet J, Reichhart JM, Hoffmann JA. Sensing and signaling during infection in Drosophila. Curr Opin Immunol 2005;17: $11-7$.

[21] Wang Z, Hu L, Yi G, Xu H, Qi Y, Yao L. ORF390 of white spot syndrome virus genome is identified as a novel anti-apoptosis gene. Biochem Biophys Res Commun 2004;325:899-907.

[22] Clem RJ, Miller LK. Control of programmed cell death by the baculovirus genes p35 and iap. Mol Cell Biol 1994;14:5212-22.

[23] Torok I, Karch F. Nucleotide sequences of heat shock activated genes in Drosophila melanogester. I. Sequences in the region of the $5^{\prime}$ and $3^{\prime}$ ends of the hsp70 gene in the hybrid plasmid 56H8. Nucleic Acids Res 1981;8:3105-23.

[24] Kozak M. Interpreting cDNA sequences: some insights from studies on translation. Mamm Genomes 1996;7:563-74.

[25] Harvey A, Soliman H, Kaiser W, Miller LK. Anti- and proapoptotic activities of baculovirus and Drosophila IAPs in an insect cell line. Cell Death Differ 1997;4:733-44.

[26] Seshagiri S, Vucic D, Lee J, Dixit VM. Baculovirus-based genetic screen for antiapoptotic genes identifies a novel IAP. J Biol Chem 1999;274:36769-73.

[27] Takramah D, Seiffert BM, Schaller M, Vigneron M, Hacker G. Baculovirus P35 interacts with a subunit of human RNA polymerase II and can enhance promoter activity in human cells. J Gen Virol 2003;84:3011-9.

[28] Vucic D, Kaiser WJ, Harvey AJ, Miller LK. Inhibition of reaperinduced apoptosis by interaction with inhibitor of apoptosis proteins (IAPs). Proc Natl Acad Sci USA 1997;94:10183-8.

[29] Vucic D, Seshagiri S, Miller LK. Characterization of reaper- and FADD-induced apoptosis in a lepidopteran cell line. Mol Cell Biol 1997; 17:667-76.

[30] Ditzel M, Wilson R, Tenev T, Zachariou Paul A, Deas E, Meier P. Degradation of DIAP1 by the N-end rule pathway is essential for regulating apoptosis. Nat Cell Biol 2003;5:467-73.

[31] Muro I, Means CJ, Clem RJ. Cleavage of the apoptosis inhibitor DIAP1 by the apical caspase DRONC in both normal and apoptotic Drosophila cells. J Biol Chem 2005;280: 18683-8.

[32] White K, Tahaoglu E, Steller H. Cell killing by the Drosophila gene reaper. Science 1996;271:805-7.

[33] McCarthy JV, Dixit VM. Apoptosis induced by Drosophila reaper and grim in a human system: attenuation by inhibitor of apoptosis proteins (clAPs). J Biol Chem 1998;273:24009-15.

[34] Bump NJ, Hackett M, Hugunin M, Seshagiri S, Brady K, Chen P, et al. Inhibition of ICE family proteases by baculovirus antiapoptotic protein p35. Science 1995;269:1885-8.

[35] Xue D, Horvitz HR. Inhibition of the Caenorhabditis elegans cell-death protease CED-3 by a CED-3 cleavage site in baculovirus p35 protein. Nature 1995;377:248-51.

[36] Zhou Q, Krebs JF, Snipas SJ, Price A, Alnemri ES, Tomaselli $\mathrm{KJ}$, et al. Interaction of the baculovirus anti-apoptotic protein $\mathrm{p} 35$ with caspases. Specificity, kinetics, and characterization of the caspase/p35 complex. Biochemistry 1998;37: 10757-65.

[37] Chai J, Du C, Wu JW, Kyin S, Wang X, Shi Y. Structural and biochemical basis of apoptotic activation by Smac/DIABLO. Nature 2000;406:855-62.

[38] Liu Z, Sun C, Olejniczak ET, Meadows RP, Betz SF, Oost T, et al. Structural basis for binding of Smac/DIABLO to the XIAP BIR3 domain. Nature 2000;408:1004-8.

[39] Hay BA, Wassarman DA, Rubin GM. Drosophila homologs of baculovirus inhibitor of apoptosis proteins function to block cell death. Cell 1995;83:1253-62.

[40] Wang SL, Hawkins CJ, Yoo SJ, Muller HA, Hay BA. The Drosophila caspase inhibitor DIAP1 is essential for cell survival and is negatively regulated by HID. Cell 1999;98:453-63.

[41] Muro I, Hay BA, Clem RJ. The Drosophila DIAP1 protein is required to prevent accumulation of a continuously generated, processed form of the apical caspase DRONC. J Biol Chem 2002;277:49644-50.

[42] Robalino J, Bartlett T, Shepard E, Prior S, Jaramillo G, Scura E, et al. Double-stranded RNA induces sequence-specific antiviral silencing in addition to nonspecific immunity in a marine shrimp: convergence of RNA interference and innate immunity in the invertebrate antiviral response? J Virol 2005;79: 13561-71.

[43] Hay BA. Understanding IAP function and regulation: a view from Drosophila. Cell Death Differ 2000;7:1045-56. 